\title{
The use of vasoconstrictors in patients with liver cirrhosis: how, when, why
}

\begin{abstract}
Portal hypertension (PH) is a severe complication of liver cirrhosis. Patients with PH run the risk of developing gastro-esophageal varices and massive gastrointestinal bleeding, ascites, hepatorenal syndrome, and hepatic encephalopathy.

Portal blood flow in its turn increases because of enhanced production of vasodilators, increased eNOS activity and NO release, systemic and splanchnic vasodilation, hyperkinetic circulation, and hyposensitivity to vasoconstrictors. Thus, it is now widely recognized that this hyperkinetic (hyperdynamic) circulation that characterizes livercirrhosis is the main cause of the complications of the disease.

This review is aimed at addressing the role of vasoconstrictor treatment in patients suffering from complications of decompensated cirrhosis, offering practical suggestions for the management of this treatment at bedside.

In particular, the management of terlipressin in patients with cirrhosis, its side effects and the efficacy of this vasoconstrictor will be examined.
\end{abstract}

Keywords: Cirrhosis; Portal hypertension; Terlipressin; Vasoconstrictors L'impiego di vasocostrittori in pazienti con cirrosi epatica: come, quando, perché CMI 2012; 6(3): 105-115

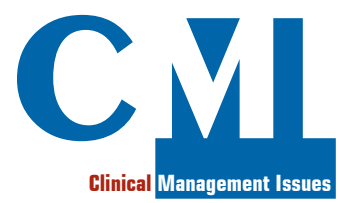

Clinical management
Department of Internal Medicine and Liver Unit, Marino General Hospital, Rome

\section{INTRODUCTION}

Portal hypertension $(\mathrm{PH})$ is a severe complication of liver cirrhosis [1]. Patients with $\mathrm{PH}$ run the risk of developing gastro-esophageal varices and massive gastrointestinal bleeding, ascites, hepatorenal syndrome, and hepatic encephalopathy [2].

The portal pressure gradient is the difference between portal pressure and the pressure at the hepatic veins/inferior vena cava level; it represents the hepatic perfusion pressure [3]. In patients with cirrhosis, portal pressure increases because of increased intrahepatic vascular resistance and increased portal blood flow [4]. The interaction between portal blood flow and the vascular resistance that opposes that flow is defined by the Ohm's law $(\Delta \mathrm{P}=\mathrm{Q} \times \mathrm{R})$, where $\Delta \mathrm{P}$ is the portal pressure gradient, $\mathrm{Q}$ is portal blood flow and $\mathrm{R}$ is the vascular resistance [5].
Increased resistance is due to:

- liver architectural disturbance, with distortion of vascular architecture by fibrosis, scarring, regenerative nodules, thrombosis (mechanical or fixed component, not modifiable by pharmacological treatment); and

- functional hepatic microcirculation alterations (active contraction of portal/septal myofibroblasts, activated stellate cells, portal venules - the so-called dynamic component, modifiable by drugs). This active intrahepatic vascular contraction is a consequence of an unbalance between vasoconstrictor substances (endothelin, angiotensin II, vasopressin, tromboxane A2, leukotrienes, etc.) and vasodilators (nitric oxide [NO], CO, prostacycline, etc.) [6]. Portal blood flow in its turn increases because of enhanced production of vasodi-
Corresponding author Claudio Puoti, MD Marino General Hospital Viale XXIV Maggio 00047 Marino, Rome Fax : +39-06-9327-3047 puoti@epatologia.org 
What is already known about this issue?

- Portal hypertension (PH) is a severe complication of liver cirrhosis. It is secondary to increased intra-hepatic resistance to portal blood flow and to massive splanchnic arteriolar vasodilation, due to an excess of NO within the splanchnic circulation

- The hyperkinetic (hyperdynamic) circulation that characterizes liver cirrhosis is the main cause of the complications of the disease, such as hepatorenal syndrome (HRS)

- HRS is a common complication of patients with cirrhosis, due to functional renal impairment without an identifiable cause

- The arterial vasodilation in the splanchnic circulation plays a central role in the renal function deterioration, with activation of renin-angiotensin and sympathetic nervous systems, leading to an uncontrolled renal vasoconstriction

lators, increased eNOS activity and $\mathrm{NO}$ release, systemic and splanchnic vasodilation, hyperkinetic circulation, and hyposensitivity to vasoconstrictors [7]. Thus, it is now widely recognized that this hyperkinetic (hyperdynamic) circulation that characterizes liver cirrhosis is the main cause of the complications of the disease [8].

This review is aimed to address the role of vasoconstrictor treatment in patients suffering from complications of decompensated cirrhosis, offering practical suggestions for the management of this treatment at bedside.

\section{PATHOPHYSIOLOGY OF PH AND HYPERKINETIC CIRCULATION}

Increased intra-hepatic resistance to portal blood flow is the primary factor leading to portal hypertension in cirrhosis [1]. As underlined above, the increased intra-hepatic resistance is the consequence of both the architectural disturbances caused by the cirrhotic process and of an active contraction of several cellular elements [8]. This latter represents the dynamic and modifiable component of intra-hepatic resistance, accounting for up to $30-40 \%$ of the intrahepatic vascular resistance in cirrhosis [9]. Contractile elements influencing the hepatic vascular bed include vascular smooth muscle cells of the intra-hepatic vasculature, activated hepatic stellate cells (HSCs) and hepatic myofibroblasts, that may compress the regenerating nodules or venous shunts within the fibrous septa [8]. Vasoactive mediators, either vasoconstrictors or vasodilators, may modulate intrahepatic vascular resistan- ce either in health or during liver disease, whatever the location where they act $[8,9]$.

In normal liver, the hepatic endothelium produces vasodilatory substances in response to increased blood volume, blood pressure, or vasoconstrictor agents, in an attempt to counteract the concomitant increase in intravascular pressure [10]. However, the cirrhotic liver cannot attenuate the increased portal blood flow caused by $\mathrm{PH}$ [3]. This socalled "endothelial dysfunction" in cirrhosis has been attributed to reduced nitric oxide (NO) bioavailability and to increased vasoconstrictor substances [8]. Such imbalance between endogenous vasoconstrictor and vasodilator factors observed in the cirrhotic liver is thought to be implicated in the pathogenesis of the dynamic component of the increased intra-hepatic resistance of the cirrhotic liver [10].

In other words, during the course of cirrhosis an excess of vasoconstrictors with respect to vasodilators does progressively occur. The main vasoconstrictors are COX1derived prostanoids, thromboxane, endothelin, angiotensin, vasopressin, and norepinephrine $[3,8,10]$.

In addition, reduced NO bioavailability within the cirrhotic liver leads to failure to counteract the increased intrahepatic vasoconstriction [11]. Endothelial NO synthase (eNOS) is responsible for most of the vascular NO produced into the normal liver [12]. By contrast, in the cirrhotic liver, there is a reduced NO bioavailability that plays a major role in increasing intra-hepatic vascular resistance and thereby worsening portal hypertension [13]. Decreased NO production occurs despite a normal expression of eNOS mRNA and normal levels of eNOS protein $[8,10]$, and has been attributed, at least in part, to reduced eNOS activity caused by several posttranslational alterations in the regulation of the enzyme such as increased caveolin expression, or a defect of the essential cofactor of eNOS (tetrahydrobiopterin), decreased eNOS phosphorylation, and increased levels of asymmetric dimethylarginine among others [10].

Finally, in patients with decompensated cirrhosis there is a massive splanchnic arteriolar vasodilation, due to an excess of $\mathrm{NO}$ within the splanchnic circulation. This vasodilation is thought to be secondary to $\mathrm{PH}$ and shear stress, that determines hyperactivation of eNOS gene within the extra-hepatic splanchnic smooth cells, with increased eNOS activity and excess NO production. 
This reduced NO availability within the intrahepatic circulation and the $\mathrm{NO}$ excess within the splanchnic extrahepatic circulation has been defined as "the paradox of NO in liver cirrhosis": too much (outside the liver), not enough (within the liver) [14].

\section{PH MEASUREMENTS}

The definition of portal hypertension is based on a pressure measurement [15]. Portal pressure measurement is usually determined indirectly, by subtracting the free hepatic venous pressure (FHVP) from the wedged hepatic venous pressure (WHVP). In liver cirrhosis, WHVP equals portal (sinusoidal) pressure, and FHVP equals inferior vena cava pressure [16]. This gradient, the so-called hepatic venous pressure gradient (HVPG), accurately reflects the degree of $\mathrm{PH}$ in the majority of liver diseases $[16,17]$. The technique of hepatic vein catheterization with measurement of the HVPG is safe and reproducible [3]. Several studies have shown a good correlation between direct portohepatic measurements and HVPG measurements (using a balloon catheter) both in alcoholic and viral cirrhosis [3]. Thus, the HVPG measurement using a balloon catheter is now considered the gold standard for portal pressure evaluation [16].

$\mathrm{PH}$ is defined by a pathological increase of the HVPG values above the normal upper limit of $5 \mathrm{mmHg}$, while clinically significant $\mathrm{PH}(\mathrm{CSPH})$ is defined by an increase in HVPG values to a threshold above approximately $10-12 \mathrm{mmHg}[2,16,17]$. Varices do not bleed when the HVPG is below 12 $\mathrm{mmHg}$ [1].

HVPG determination is a safe and reliable tool to measure the degree of portal hypertension, with a very low rate of complications. However, it is an invasive technique, not inexpensive, which requires well-experienced hepatologists, specific equipments and expensive disposable materials [18].

Thus, due to these methodological and technical difficulties, the measurement of $\mathrm{PH}$ is not immediate and is performed only in a limited number of specialized centres [19].

In clinical practice, HVPG measurement could have several applications [20-23], such as:

- Evaluation of the risk of variceal haemorrhage.
- Assessment of haemodynamic response to pharmacological therapy.

- Definition of prognosis (cirrhosis and acute variceal bleeding).

- Pre-operative evaluation of cirrhotic patients candidates to hepatic resection.

Furthermore, HVPG measurement could have a prognostic value. It has been shown that the HVPG at different cut-off levels is a predictor of long-term survival in cirrhotic patients without previous variceal bleeding at inclusion in the study [24]. Several studies have found a significant higher survival in patients in whom HVPG levels were below the cut-off than in those with HVPG above the cut-off [25]. The predictive HVPG value was identified between 12 and $20.8 \mathrm{mmHg}$. Further, it has been found that early measurement of HVPG in patients with acute variceal bleeding could have a negative prognostic value if $\geq 20 \mathrm{mmHg}[26,27]$.

\section{PHARMACOLOGICAL PROFILE OF VASOCONSTRICTORS}

In clinical practice, at least four vasoconstrictors are used: somatostatin, octreotide, terlipressin and midodrine.

Somatostatin (SMS) is a 14 -aminoacid peptide, with well-known vasoconstrictive properties. SMS provokes splanchnic vasoconstriction with a decrease in portal blood flow, acting through inhibition of endogenous vasodilators (glucagon, VIP, P substance), although it exerts also a direct vasoconstrictive effect, mediated by activation of receptor subtype 2. Although SMS treatment is generally safe and well tolerated, in some patients several side effects are seen. The main reported side effects of SMS are: hyper/hypoglycaemia, diarrhoea, hypertension, chest pain, flushing [28].

Octreotide (OCT) is a synthetic 8-peptide, sharing with SMS the 4 aminoacids responsible for biological effects. Reported side effects of OCT are: dizziness, fatigue, headache, diarrhoea, abdominal pain, flushing, nausea [8].

On the whole, SMS and OCT have a lower incidence of complications, major adverse events (AEs) and mortality than vasopressin and terlipressin.

Terlipressin (triglycyl-lysine-vasopres$\sin$ ) is a synthetic analogue of the natural hormone arginin-vasopressin. Terlipressin is the most used drug in the treatment of 
hepatorenal syndrome (HRS). Terlipressin is a prohormone of lysine-vasopressin (triglycyl-lysine-vasopressin). Following intravenous administration, the glycyl residues are cleaved from the prohormone by endothelial peptidases, allowing prolonged release of lysine-vasopressin. This mechanism prolongs the half-life of terlipressin, enabling administration in divided doses without the need for an infusion as with vasopressin and minimizes systemic toxicity [29].

Terlipressin has affinity for both V1 and V2 receptors. Terlipressin selectively causes splanchnic and extrarenal vasoconstriction by stimulation of $\mathrm{V} 1$ receptors, which are predominantly located in the smooth muscles of the arterial vasculature in the splanchnic region, and thereby reduces splanchnic blood flow and portal pressure [30]. Following terlipressin administration for 30 minutes there is an increase in mean arterial pressure and systemic vascular resistance while the heart rate, cardiac output, hepatic venous pressure gradient, and portal venous blood flow decrease [31].

Reduction in portal pressure results in amelioration in the hyperdynamic circulation, thereby improving the effective circulatory volume, and renal perfusion pressure [32].

V2 receptor stimulation by terlipressin increases water reabsorption in the renal collecting ducts by increasing the number of aquaporin-2 water channels in the apical plasma membrane $[14,16]$. Hyponatraemia may occur in some patients [33].

Due to prolonged vasoconstrictor action terlipressin can be given by intermittent i.v. injections (preferably in bleeding) or by continuous infusion (HRS).

Although terlipressin is slowly cleaved in vivo to vasopressin, it is also believed to have an intrinsic vasoconstrictor effect on its own. Terlipressin has a much greater effect on vascular vasopressin receptors (V1) than on renal vasopressin receptors (V2) [34].

Midodrine is an alpha1-adrenergic agonist [29]. Data supporting the use of midodrine were mostly observational in nature and have not been compared directly to treatment with terlipressin or noradrenaline. Oral administration of this alpha-adrenergic agonist improved systemic and renal haemodynamics in nonazotaemic cirrhotic patients but had no effect in patients with HRS [30]. However, when midodrine was combined with plasma volume expansion and octreotide, a nonspecific inhibitor of the release of endogenous vasodilators, there was significant improvement in both the systemic and renal haemodynamics and urinary sodium excretion, although renal function did not return to normal despite suppression of all measured neurohormonal systems to within the normal range [33].

\section{THE ROLE OF VASOCONSTRICTORS IN THE TREATMENT OF COMPLICATION OF CIRRHOSIS}

\section{Gastroesophageal varices}

Gastroesophageal varices are present in almost half of patients with cirrhosis at the time of diagnosis, with the highest rate among patients with Child-TurcottePugh class B or C [35]. Development and growth of gastroesophageal varices each occur at a rate of $7 \%$ per year [1]. The 1-year rate of a first variceal haemorrhage is approximately $12 \%$ ( $5 \%$ for small varices and $15 \%$ for large varices) [35]. Besides variceal size, red wale marks on varices and advanced liver disease (Child class B or C) identify patients at a high risk for variceal haemorrhage [1].

The 1-year rate of recurrent variceal haemorrhage is approximately $60 \%$ [35]. The 6-week mortality with each episode of variceal haemorrhage is approximately 15 to $20 \%$, ranging from $0 \%$ among patients with Child class A disease to approximately 30\% among patients with Child class C disease [1,2].

Available therapies for varices and variceal haemorrhage can be classified according to whether they act on the physiologic mechanisms of portal hypertension [36].

Acute variceal bleeding is the most severe consequence of portal hypertension. The treatment of this entity should involve the initial control of haemorrhage and prevention of early rebleeding [37]. Endoscopic sclerotherapy or band ligation have been shown to be highly effective in the control of both these processes [38]. Both procedures stop bleeding in about $80 \%$ to $90 \%$ of patients [1]. On the other hand, vasoactive drugs, such as somatostatin and terlipressin, are as effective as endoscopic sclerotherapy for the arrest of the acute episode of bleeding and prevention of early rebleeding [39]. However, haemodynamic and clinical data 
are not so consistent when other agents such as octreotide are employed [40].

Patients who have Child class A or B disease or who have an HVPG of less than 20 $\mathrm{mmHg}$ have a low or intermediate risk and should receive standard therapy - specifically, the combination of a safe vasoconstrictor (terlipressin, somatostatin, or analogues such as octreotide or vapreotide, administered from the time of admission and maintained for 2 to 5 days) and endoscopic therapy (preferably endoscopic variceal ligation, performed at diagnostic endoscopy $<12$ hours after admission), together with short-term prophylactic antibiotics (either norfloxacin or ceftriaxone) [41].

A recent metanalysis showed that the efficacy of endoscopic therapy in achieving initial control of bleeding and 5-day haemostasis is significantly improved when somatostatin or its derivatives are added to the endoscopic treatment regimen [42].

\section{Hepatorenal syndrome (HRS)}

HRS is a common complication of patients with cirrhosis, due to functional renal impairment without an identifiable cause $[43,44]$. Approximately $39 \%$ of patients with cirrhosis and ascites will develop HRS during the course of the disease. Criteria for the diagnosis of HRS developed by the International Club of Ascites [45] include the following: presence of cirrhosis and ascites, serum creatinine higher than $1.5 \mathrm{mg} / \mathrm{dl}$, no improvement in serum creatinine (decrease equal to or less than $1.5 \mathrm{mg} / \mathrm{dl}$ ) after at least 48 hours of diuretic withdrawal and volume expansion with albumin, absence of shock, no current or recent treatment with nephrotoxic drugs, absence of parenchymal kidney disease as indicated by proteinuria (500 mg/day), microhaematuria (50 red blood cells/high power field), and/or abnormal renal ultrasound scanning. Type $1 \mathrm{HRS}$ was defined as a rapidly progressive reduction in renal function, e.g., a doubling of serum creatinine to greater than $2.5 \mathrm{mg} / \mathrm{dl}$ in less than 2 weeks and failure of renal function to improve following diuretic withdrawal and plasma volume expansion. Type 2 HRS was defined as serum creatinine greater than $1.5 \mathrm{mg} / \mathrm{dl}$, which follows a steady or slowly progressive course [46].

Again, HRS pathogenesis involves the interplay between the activation of vasoconstrictor systems and the reduction in the activity of vasodilator systems [44].
The arterial vasodilation in the splanchnic circulation plays a central role in the renal function deterioration and the haemodynamic changes and is mediated by an increased production and/or activity of local vasodilators, with nitric oxide being the most important. As the liver disease progresses in severity, a critical level of hypoperfusion is achieved, with subsequent activation of renin-angiotensin and sympathetic nervous systems [47].

Renal vasodilatory systems are not able to offset the maximal effect of endogenous vasoconstrictors, leading to an uncontrolled renal vasoconstriction $[2,4,5]$. This peripheral arterial vasodilation theory for HRS has resulted in several pharmacologic treatment of HRS with systemic vasoconstrictors [10,11]. Preliminary studies have been reported using $\alpha$-adrenergic agonists (midodrine and noradrenaline), vasopressin analogues (ornipressin and terlipressin), and somatostatin analogue (octreotide) [12,13]. Octreotide in combination with midodrine and albumin infusion improved renal and systemic haemodynamics by the systemic vasoconstrictor effect of midodrine and the inhibition of endogenous vasodilator release action of octreotide [14].

The choice of drug and the schedule of treatment varied across these studies. To date, terlipressin is the most widely used vasoconstrictor in the treatment of type-1 HRS [48,49].

Partial or complete reversal of type- 1 HRS was observed in almost 59\% of patients $[50,51]$. Nevertheless, the preliminary results of the two first controlled clinical trials comparing terlipressin and albumin with albumin alone did not confirm a beneficial effect of terlipressin and albumin on 2- or 3 -month survival in patients with type-1 HRS [50,51].

More recently, Caraceni et al. suggested long-term treatment with terlipressin plus albumin (62 days to eight months) as a bridge to liver transplantation in cirrhotic patients with recurrent HRS [52].

In clinical practice, Italian regulatory Agency suggests the use of terlipressin in cirrhotic patients with HRS type I, provided that they fulfil the criteria for the diagnosis of HRS as defined by the International Ascites Club [45]; terlipressin should be administered at a dosage of 2-3 mg/die by continuous infusion or by intermittent i.v. boli of $0.5 \mathrm{mg}$ every 4-6 hours. 
Which are the neru findings?

- Type-1 HRS is a life-threatening complications of cirrhosis, with high mortality rates in the short term period

- The use of vasoconstrictors in combination with plasma expanders (terlipressin plus albumin) seems to be able to reverse rapidly progressive renal failure

- Partial or complete reversal of type-1 HRS was observed in up to 60\% of patients receiving treatment with terlipressin plus albumin, and more recent data suggest that terlipressin seems to be able to reduce mortality in these subjects

- Results with other vasoconstrictors are still preliminary and conflicting
The first-line treatment of refractory ascites consists in repeated total paracenteses [56]. In the case of exceedingly high frequency of paracentesis, the use of transjugular intrahepatic portosystemic shunts (TIPS) should be taken into consideration [61]. Patients may continue to receive diuretic treatment, if tolerated.

Terlipressin has been proposed for the treatment of refractory ascites, in association with the prolonged infusion of human albumin, but to date its use in cirrhotic patients with refractory ascites and normal renal function should be considered still preliminary [62]. A recent study [63] showed a synergistic effect of terlipressin when added to albumin and diuretics in patients with refractory ascites and normal renal function, suggesting that albumin might enhance the vasoconstrictive response to terlipressin. This might contribute to counterbalance the negative effects of systemic vasodilation, which characterize the hyperdynamic circulation of cirrhotic patients [63]. liver cirrhosis, occurring in more than 50 $60 \%$ of the patients within 10 years of the diagnosis. It develops late during the course of the disease, when there are severe portal hypertension and hepatic insufficiency; its appearance represents a critical landmark in the natural history of the disease, with a significant worsening of the prognosis [56].

Furosemide and spironolactone - together with salt restriction - are the diuretics more commonly used in the treatment of mild to moderate cirrhotic ascites [57] Furosemide inhibits chloride and sodium reabsorption in the thick ascending limb of the loop of Henle, but has no effect on the distal nephron. Spironolactone undergoes extensive metabolism leading to numerous biologically active compounds, the most important quantitatively being canrenone. In the kidney, these compounds act by competitively inhibiting the tubular effect of aldosterone in the distal nephron [58].

In $5-10 \%$ of patients, ascites cannot be mobilized, or its early recurrence (e.g. after therapeutic paracentesis) cannot be prevented by medical treatment. This condition is known as "refractory ascites" [59]. This condition might be secondary to the lack of response to sodium restriction and maximal diuretic treatment $(160 \mathrm{mg} /$ day of furosemide and $400 \mathrm{mg} /$ day of spironolactone - diuretic-resistant ascites) or to the development of diuretic-induced complications that might preclude the use of an effective diuretic dosage (diuretic-intractable ascites) [60].

\section{MANAGEMENT OF SIDE EFFECTS OF VASOCONSTRICTORS}

In everyday clinical practice the most used vasoconstrictors in cirrhotic patients are somatostatin and terlipressin. The use of SMS is limited to the control of acute variceal bleeding, whilst terlipressin treatment is employed for the management of several complications of cirrhosis: acute variceal bleeding, HRS type I, refractory ascites with normal renal function.

However, while adverse events during treatment with SMS are rather rare and usually not severe (hyper/hypoglycaemia, diarrhoea, hypertension, chest pain, flushing), side effects of terlipressin are much more frequent, more severe, more difficult to manage, and - although rarely - even life threatening [29-33].

A randomized prospective double-blind, placebo-controlled trial of terlipressin for type I HRS [50] showed an higher incidence of severe adverse events up to 30 days posttreatment in patients treated with terlipres$\sin (8.9 \%$ vs. $1.8 \%)$, with a rate of withdrawal due to adverse events (AEs) up to 7 days of $5.4 \%$ vs. $0 \%$. In another patients' series, there were $4 \%$ of myocardial infarction, $13 \%$ of intestinal ischaemia, $9 \%$ of arrhythmias, and $30 \%$ of circulatory overload [51].

Due to its ADH-like action (argininvasopressin effects), terlipressin might in- 
duce severe hyponatraemia, that might be misinterpreted as hepatic encephalopathy. Solà et al. [64] found that the development of hyponatraemia was common in cirrhotic patients treated with terlipressin because of GI bleeding. During the 5-days treatment period, $67 \%$ of the patients developed acute reduction in serum sodium concentrations, which was marked (reduction $>10 \mathrm{mEq} / \mathrm{l}$ ) in up to $36 \%$ of them, and associated with neurological manifestations in some patients. Similar findings were reported by Krag and coworkers [65].

As to cardiovascular adverse events, terlipressin treatment might induce myocardial infarction [66], severe bi-ventricular dysfunction [67], and Tako-Tsubo syndrome (also known as transient left ventricular apical ballooning syndrome), a clinical entity characterized by reversible left ventricular apical wall motion abnormalities, typical electrocardiographic features, and minor increase of serum heart enzymes [68].

Other relevant side effects, sometimes severe, are peripheral ischemia [69], cutaneous necrosis $[70,71]$, haemorragic blistering of the skin [72], and even severe extensive epidermal necrosis [73].

In Table I some advice are listed in order to avoid and diagnose the adverse events caused by terlipressin.
The main adverse events during terlipressin therapy

- Myocardial ischaemia or infarction, cardiac arrbythmias

- Arterial hypertension

- Severe hyponatraemia, seizures, loss of consciousness

- Pulmonary hypertension, dyspnoea

- Headache

- Abdominal pain, intestinal ischaemia or infarction

- Peripheral ischaemia

- Acute vasculitis-like lesions

- Raynaud's phenomenon

- Peripheral tissue necrosis

Predictors of adverse events during terlipressin

- Elderly

- Male gender

- Coronary heart disease

- Arterial hypertension

- History of arrhytmias

- Illicit drug abuse (cocaine)

- Heavy smokers

- Obesity

- Chronic obstructive pulmonary disease (COPD)

- Previous Raynaud's phenomenon

- Spontaneous bacterial peritonitis (SBP)

- Peripheral venous insufficiency

\section{Timing as to treatment $\quad$ Advice to manage terlipressin adverse events}

\begin{tabular}{|l|ll} 
Before & $\begin{array}{l}\text { 1. } \\
\text { 2. }\end{array}$ Use terlipressin cautiously in patients with known coronary heart \\
disease or history of arrhytmias
\end{tabular}

Table I. How to manage terlipressin adverse events 
Which are the implications in clinical practice?

- Side effects of terlipressin are more severe and more difficult to manage than those of other vasoconstrictors, and - although rarely - even life threatening

- Hyponatraemia and acute ischaemia (heart, gut, skin, etc.) have been frequently reported

- Anyway, the safety profile is favourable when considering the clinical efficacy and the high mortality caused by the complications of cirrhosis

- Among available vasoactive drugs, terlipressin is probably the most effective pharmacological therapy for bleeding and the only accepted treatment for HRS, but SMS appears to be also effective with less side effects in variceal bleeding

\section{CONCLUSIONS}

Despite these adverse events, it should be underlined that terlipressin decreases failure of initial haemostasis by $34 \%$, decreases mortality by $34 \%$, and is considered a firstline treatment for esophageal bleeding [74]. Terlipressin in combination with albumin reverses type $1 \mathrm{HRS}$ in 33-60\% of cases and is the only treatment with proven efficacy in randomized trials [75].

Thus, we can conclude that the safety profile is favourable when considering the clinical efficacy and the high mortality caused by the complications of cirrhosis. Mortality and withdrawal of terlipressin due to adverse events occurs in less than $1 \%$ of cases. Mild adverse events related to terlipressin treatment occur in 10-20\% of patients.

Among available vasoactive drugs, terlipressin is probably the most effective pharmacological therapy for bleeding and the only accepted treatment for HRS, but SMS appears to be also effective with less side effects in variceal bleeding [1].

\section{DISCLOSURE}

The author declares no conflict of interest.

\section{REFERENCES}

1. de Franchis R. Revising consensus in portal hypertension: report of the Baveno V consensus workshop on methodology of diagnosis and therapy in portal hypertension. J Hepatol 2010; 53: $762-8$

2. Bosch J, Abraldes JG, Groszmann R. Current management of portal hypertension. J Hepatol 2003; 38: S54-S58

3. Thalheimer U, Bellis L, Puoti C, et al. Should we routinely measure portal pressure in patients with cirrhosis, using hepatic venous pressure gradient (HVPG) as a guide for prophylaxis and therapy of bleeding and rebleeding? Eur J Intern Med 2011; 22: 5-7

4. Burroughs AK, Thalheimer U. Hepatic venous pressure gradient in 2010: optimal measurement is key. Hepatology 2010; 51: 1894-6

5. Thalheimer U, Leandro G, Mela M, et al. Systematic review of HVPG measurement: statistics versus clinical applicability. Gastroenterology 2007; 132: 1201-2

6. Triantos CK, Nikolopoulou V, Burroughs AK. The therapeutic and prognostic benefit of portal pressure reduction in cirrhosis. Aliment Pharmacol Ther 2008; 28: 943-52

7. Bosch J, Abraldes JG, Berzigotti A, et al. The clinical use of HVPG measurements in chronic liver disease. Nat Rev Gastroenterol Hepatol 2009; 6: 573-82

8. Bosch J, Abraldes JC, Fernàndez M, et al. Hepatic endothelial dysfunction and abnormal angiogenesis: new targets in the treatment of portal hypertension.J Hepatol 2010; 53: 558-67

9. Bellis L, Castellacci R, Puoti C. Hepatic venous pressure gradient determination in patients with hepatitis C virus-related and alcoholic cirrhosis Eur J Gastroenterol Hepatol 2003; 15: 1085-9

10. Bosch J, Berzigotti A, Garcia-Pagan JC, et al. The management of portal hypertension: rational basis, available treatments and future options.J Hepatol 2008; 48: S68-S92

11. Hernandez-Guerra M, Garcia-Pagan JC, Bosch J. Increased hepatic resistance: a new target in the pharmacologic therapy of portal hypertension.J Clin Gastroenterol 2005; 39: S131-S137 
12. Fernandez M, Semela D, Bruix J, et al. Angiogenesis in liver disease.J Hepatol 2009; 50: 604-20

13. Shah V,Toruner M,Haddad F, et al. Impaired endothelial nitric oxide synthase activity associated with enhanced caveolin binding in experimental cirrhosis in the rat. Gastroenterology 1999; 117: 1222-8

14. Wiest R, Groszmann RJ. The paradox of nitric oxide in cirrhosis and portal hypertension: too much, not enough. Hepatology 2002; 35: 478-81

15. Parikh S. Hepatic venous pressure gradient: worth another look? Dig Dis Sci 2009; 54: 1178-83

16. Perellò A, Escorsell A, Bru C, et al. Wedged hepatic venous pressure adequately reflects portal pressure in Hepatitis C virus-related cirrhosis. Hepatology 1999; 30: 1393-7

17. Bellis L, Puoti C. Hepatic haemodynamic: research activity or clinical tool? Ital J Med 2010; 4: 201-6

18. Kumar A, Sharma P, Sarin SK. Hepatic venous pressure gradient measurement: time to learn! Indian J Gastroenterol 2008; 27: 74-80

19. Groszmann RJ, Wongcharatrawee S. The hepatic venous pressure gradient: anything worth doing should be done right. Hepatology 2004; 39: 280-2

20. Escorsell A, Bordas JM, Castaneda B, et al. Predictive value of the variceal pressure response to continued pharmacological therapy in patients with cirrhosis and portal hypertension. Hepatology 2000; 31: 1061-7

21. Triantos CK, Nikolopoulou V, Burroughs AK. The therapeutic and prognostic benefit of portal pressure reduction in cirrhosis. Aliment Pharmacol Ther 2008; 28: 943-52

22. Bruix J, Castells A, Bosch J, et al. Surgical resection of hepatocellular carcinoma in cirrhotic patients: prognostic value of preoperative portal pressure. Gastroenterology 1996; 111: 1018-22

23. Sanyal AJ. Hepatic Venous Pressure Gradient: to measure or not to measure, that is the question. Hepatology 2000: 31: 1175-6

24. Puoti C, Bellis L. Steatosis and portal hypertension. Eur Rev Med Pharmacol Sci 2005; 9:285-90

25. Hicken BL, Sharara AI, Abrams GA, et al. Hepatic venous pressure gradient measurements to assess response to primary prophylaxis in patients with cirrhosis: a decision analytical study. Aliment Pharmacol Ther 2003; 17: 145-53

26. Bosch J, Garcia-Pagan JC. Prevention of variceal rebleeding. Lancet 2003; 361: 952-4

27. Vorobioff JD. Hepatic venous pressure in practice. How, when and why. J Clin Gastroenterol 2007; 41: S336-S343

28. de Franchis R, Dell'Era A, Iannuzzi F. Diagnosis and treatment of portal hypertension. Digest Liver Dis 2004; 36: 787-98

29. Sarin SK, Sharma P. Terlipressin: an asset for hepatologists. Hepatology 2011; 54: 724-8

30. Moreau R, Lebrec D. The use of vasoconstrictors in patients with cirrhosis: type $1 \mathrm{HRS}$ and beyond. Hepatology 2006; 43: 385-94

31. Moller S, Hansen EF, Becker U, et al. Central and systemic hemodynamic effects of terlipressin in portal hypertensive patients. Liver 2000; 20: 51-9

32. Narahara Y, Kanazawa H, Taki Y, et al. Effects of terlipressin on systemic, hepatic and renal hemodynamics in patients with cirrhosis. J Gastroenterol Hepatol 2009; 24: 1791-7

33. Dobre M, Demirjian S, Sehgal AR. Terlipressin in hepatorenal syndrome: a systematic review and meta-analysis. Int Urol Nephrol 2011; 43: 175-84

34. Obritsch MD, Bestul DJ, Jung R, et al. The role of vasopressin in vasodilatory septic shock. Pharmacotherapy 2004; 24: 1050-63

35. Garcia-Tsao G, Sanyal AJ, Grace ND. Prevention and management of gastroesophageal varices and variceal hemorrhage in cirrhosis. Hepatology 2007; 46: 922-38

36. D'Amico G, Pagliaro L, Bosch J. Pharmacological treatment of portal hypertension: an evidencebased approach. Semin Liver Dis 1999; 19: 475-505

37. D'Amico G, de Franchis R. Upper digestive bleeding in cirrhosis. Post therapeutic outcome and prognostic indicators. Hepatology 2003; 38: 599-612

38. Carbonell N, Pauwels A, Serfaty L, et al. Improved survival after variceal bleeding in patients with cirrhosis over the past two decades. Hepatology 2004; 40: 652-9

39. Baik SK, Jeong PH, Ji SW, et al. Acute hemodynamic effects of octreotide and terlipressin in patients with cirrhosis: a randomized comparison. Am J Gastroenterol 2005; 100: 631-5

40. Corley DA, Cello JP, Adkisson W, et al. Octreotide for acute esophageal variceal bleeding: a meta-analysis. Gastroenterology 2001; 120: 946-54

41. Garcia-Tsao G, Bosch J. Management of varices and variceal hemorrhage in cirrhosis. N Engl JMed 2010; 362: 823-32 
42. Banares R, Albillos Agustìn, Rincon D, et al. Endoscopic treatment versus endoscopic plus pharmacologic treatment for acute variceal bleeding: a meta-analysis. Hepatology 2002; 35: 609-15

43. Solà $\mathrm{E}$, Ginès P. Renal and cyrculatory dysfunction in cirrhosis: current management and future perspectives.J Hepatol 2010; 53: 1135-45

44. Wong F, Blendis L. New challenge of hepatorenal syndrome: prevention and treatment. Hepatology 2001; 34: 1242-51

45. Angeli P, Merkel C. Pathogenesis and management of hepatorenal syndrome in patients with cirrhosis. J Hepatol 2008; 48: S93-S103

46. Salerno F, Gerbes A, Gines P, et al. Diagnosis, prevention and treatment of the hepato-renal syndrome in cirrhosis. Gut 2007; 56: 1310-8

47. Angeli P, Fernandez-Varo G, Dalla Libera V, et al. The role of nitric oxide in the pathogenesis of systemic and splanchnic vasodilation in cirrhotic rats before and after the onset of ascites. Liver 2005; 29: 429-37

48. Halimi C, Bonnard P, Bernard B, et al. Effect of terlipressin (glypressin) on hepato-renal syndrome in cirrhotic patients: results of a multicenter pilot study. Eur J Gastroenterol Hepatol 2002; $14: 153-8$

49. Mulkay JP, Louis H, Donckier V, et al. Long-term terlipressin administration improves renal function in cirrhotic patients with type 1 hepato-renal syndrome: a pilot study. Acta Gastroenterol Belg 2001; 64: 15-9

50. Sanyal A, Boyer T, Garcia-Tsao G, et al. A prospective randomized double blind placebo controlled trial of terlipressin for type 1 hepato-renal syndrome (HRS). Gastroenterology 2008; 134: $1360-8$

51. Martin-Llahi M, Pepin MN, Guevara M, et al. Randomized comparative study of terlipressin and albumin vs albumin alone in patients with cirrhosis and hepato-renal syndrome. $G$ astroenterology 2008; 134: 1352-9

52. Caraceni P, Santi L, Mirici F et al. Long-term treatment of hepatorenal syndrome as a bridge to liver transplantation. Digest Liver Dis 2011; 43: 242-5

53. Esrailian E, Pantangco ER, Kyulo NL, et al. Octreotide/Midodrine therapy significantly improves renal function and 30-day survival in patients with type 1 hepatorenal syndrome. Dig Dis Sci 2007; 52: 742-8

54. Kalambokis G, Economou M, Fotopoulos A et al. The effects of chronic treatment with octreotide versus octreotide plus midodrine on systemic hemodynamics and renal hemodynamics and function in nonazotemic cirrhotic patients with ascites. Am J Gastroenterol 2005; 100: 879-85

55. Sharma P, Kumar A, Shrama BC, et al. An open label, pilot, randomized controlled trial of noradrenaline versus terlipressin in the treatment of type 1 hepatorenal syndrome and predictors of response. Am J Gastroenterol 2008; 103: 1689-97

56. European Association for the Study of the Liver. EASL clinical practice guidelines on the management of ascites, spontaneous bacterial peritonitis, and hepatorenal syndrome in cirrhosis. J Hepatol 2010; 53: 397-417

57. Runyon BA. American Association for the Study of the Liver (AASLD) Practice Guidelines. Management of adult patients with ascites due to cirrhosis: an update. Hepatology 2009; 49: 2087-107

58. Angeli P, Fasolato S, Mazza E, et al. Combined versus sequential diuretic treatment of ascites in nonazotemic patients with cirrhosis: results of an open randomized clinical trial. Gut 2010; 59: $98-104$

59. Salerno F, Guevara M, Bernardi M, et al. Refractory ascites: pathogenesis, definition and therapy of a severe complication in patients with cirrhosis. Liver Int 2010; 30: 937-47

60. Spahr L, Villeneuve JP, Tran HK, et al. Furosemide-induced natriuresis as a test to identify cirrhotic patients with refractory ascites. Hepatology 2001; 33: 28-31

61. Albillos A, Bañares R, Gonzáles $\mathrm{M}$, et al. A meta-analysis of transjugular intrahepatic portosystemic shunt versus paracentesis for refractory ascites. J Hepatol 2005; 43: 990-6

62. Krag A, Møller S, Henriksen JH. Terlipressin improves renal function in patients with cirrhosis and ascites without hepatorenal syndrome. Hepatology 2007; 46: 1863-71

63. Fimiani B, Della Guardia D, Puoti C, et al. The use of terlipressin in cirrhotic patients with refractory ascites and normal renal function: a multicentric study. Eur J Intern Med 2011; 22: $587-90$

64. Solà E, Lens S, Guevara M, et al. Hyponatremia in patients treated with terlipressin for severe gastrointestinal bleeding due to portal hypertension. Hepatology 2010; 52: 1783-90 
65. Krag A, Moller S, Bendtsen F. Hyponatremia in patients treated with terlipressin: mechanisms and implications for clinical practice. Hepatology 2011; 53: 368-9

66. Lee MY, Chu CS, Lee KT, et al. Terlipressin-related acute myocardial infarction: a case report and literature review. KJ Med Sci 2004; 20: 604-8

67. Elzouki AN, El-Menyar A, Ahmed E, et al. Terlipressin-induced severe left and right ventricular dysfunction in patient presented with upper gastrointestinal bleeding: case report and literature review. Am J Emerg Med 2010; 28: 541-6

68. Di Micoli A, Buccione D, Degli Esposti D, et al. Terlipressin infusion induces Tako-Tsubo sindrome in a cirrhotic man with hepato-renal sindrome. Intern Emerg Med 2011; 6: 437-40

69. Bañuelos Ramírez DD, Sánchez Alonso S, Ramírez Palma MM. Sildenafil in severe peripheral ischemia induced by terlipressin. A case report. Reumatol Clin 2011; 7: 59-60

70. Posada C, Feal C, García-Cruz A, et al. Cutaneous necrosis secondary to terlipressin therapy. Acta Derm Venereol 2009; 89: 434-5

71. Di Micoli A, Bracci E, Mirici Cappa F, et al. Terlipressin infusion induces ischemia of breast skin in a cirrhotic patient with hepatorenal syndrome. Digest Liver Dis 2008; 40: 304-5

72. Donnellan F, Cullen G, Hegarty JE, et al. Ischaemic complications of glypressin in liver disease: a case series. Br J Clin Pharmacol 2007; 64: 550-2

73. Yefet E, Gershovich M, Farber E, et al. Extensive epidermal necrosis due to terlipressin. Isr Med Assoc J 2011; 13: 180-1

74. Krag A, Borup T, Moller S, et al. Efficacy and safety of terlipressin in cirrhotic patients with variceal bleeding or hepatorenal syndrome. Adv Ther 2008; 25: 1105-40

75. Gluud LL, Christensen K, Christensen E, et al. Terlipressin for hepatorenal syndrome. Cochrane Database Syst Rev 2012; 9: CD005162 